\title{
RoadRunneR: A Small And Fast Bitslice Block Cipher For Low Cost 8-bit Processors
}

\author{
Adnan Baysal ${ }^{1,2}$ and Sühap Şahin ${ }^{2}$ \\ 1 TÜBİTAK BİLGEM, Gebze Kocaeli 41470, Turkey \\ 2 Department of Computer Engineering, Kocaeli University, \\ Umuttepe Yerleşkesi Kocaeli 41380, Turkey
}

\begin{abstract}
Designing block ciphers targeting resource constrained 8-bit CPUs is a challenging problem. There are many recent lightweight ciphers designed for better performance in hardware. On the other hand, most software efficient lightweight ciphers either lack a security proof or have a low security margin. To fill the gap, we present RoadRunneR which is an efficient block cipher in 8-bit software, and its security is provable against differential and linear attacks. RoadRunneR has lowest code size in Atmel's ATtiny45, except NSA's design SPECK, which has no security proof. Moreover, we propose a new metric for the fair comparison of block ciphers. This metric, called $S T / A$, is the first metric to use key length as a parameter to rank ciphers of different key length in a fair way. By using $S T / A$ and other metrics in the literature, we show that RoadRunneR is competitive among existing ciphers on ATtiny45.
\end{abstract}

Keywords: lightweight, cryptography, block cipher, bitslice, 8-bit CPU, Wireless Sensor Network, ATtiny 45

\section{Introduction}

As the price of small electronic devices decreases, notions like ubiquitous computing, Internet of things, and smart buildings become more popular each day. RFID tags and low cost 8-bit CPUs are commonly deployed in these applications. Atmel's ATtiny45, one of commonly used 8-bit CPUs, costs less than $\$ 1$ [1]. This availability and programmable nature make these CPUs a good choice for many applications such as wireless sensor networks (WSNs).

One of the main problems in such applications is the security and privacy of information shared between devices. In many applications, data is shared between the nodes and the server over the air. Hence, an attacker can possibly get private information, or even change it for her/his benefit. For this reason, it is required to use cryptographic algorithms in these applications. Since the nodes are resource constrained in terms of memory, frequency and energy, use of lightweight cryptography becomes the best option in these applications.

Block ciphers are one of the main primitives for cryptographic applications. Therefore, the design of lightweight block ciphers has attracted many researchers' attention, especially in the last 10 years. There are many designs, some with 
innovative ideas, such as LBlock [45], LED [26], PRESENT [10], PRINCE [12], PRINTCIPHER [32], SEA [41], TEA [44], SIMON and SPECK [5], ITUbee [28], PRIDE [3], and RECTANGLE [47]. Most of these algorithms used building blocks to optimize hardware implementations. For this reason, many of these algorithms are not a good choice for software applications in 8-bit CPUs. On the other hand, some more recent designs such as ITUbee, SPECK, PRIDE, and RECTANGLE are focused on performance in software to be an alternative in low cost CPUs. Some of these recent ciphers use bitslice substitution layers (Sboxes) where Boolean operations on CPU words are used to describe the S-box. By this approach, look-up-tables can be avoided which saves code size and CPU clock cycles. Moreover, since bitslice ciphers use small S-boxes, their hardware areas are small.

Another problem in block cipher design is the comparison of efficiencies of different ciphers for an application, and sometimes for academic purposes. Each platform and application has its own constraints and a simple comparison of area or throughput values are neither enough nor fair. Formulas for ranking block ciphers using the area-speed characteristics are needed, since implementation methods affect both values.

$\frac{\text { Throughput }}{\text { area }}$ is one metric offered in [11] to make a fair comparison of block ciphers in different hardware implementation methods (serial, parallel, pipe-lined, etc.). Badel et al. [4] expanded this definition by considering the possibility to trade-off throughput for power in energy-critical applications. Their formula is called Figure Of Merit (FOM) and defined as $\frac{\text { Throughput }}{\text { Area }^{2}}$. This formula is further improved by Khoo et al. [29] by calculating throughput at the minimum round number that the cipher is secure according to a security metric, and called this comparison metric as Figure Of Adversarial Merit (FOAM). In their paper, this security metric is the number of active S-boxes in differential and linear trails.

In [20], a new definition of FOM for software implementations was given. In that paper, authors suggested summing each performance indicator (code size, ram size, cycle counts) divided by the minimum of that value in the compared ciphers in a weighted manner, i.e., by multiplying each indicator with its corresponding weight. In this approach, hardest part is to find reasonable and useful weights, and they selected all weights as 1 . None of the metrics above use key size in their formula. Therefore, there is no fair way of comparing ciphers of different key sizes using the metrics in the literature.

\subsection{Our contribution}

We designed a new lightweight block cipher, RoadRunneR, with the goal of efficiency (especially in 8-bit low cost CPUs) and provable security in terms of minimum number of active S-boxes in differential and linear trails. The cipher is especially designed to have a very low code size, while having high throughput. Simulation results showed that on ATtiny45, our cipher have the least code size among other compared lightweight ciphers, except NSA's design SPECK which has no provable security properties to determine the round number. Our preliminary cryptanalysis showed that RoadRunneR have a relatively high security 
margin in contrast to most lightweight ciphers. RoadRunneR has variable areatime-security trade-off characteristics with different implementation methods so that it can fit the needs of specific application it may be used in.

Moreover, we defined a new efficiency comparison metric for block ciphers which (to the best of our knowledge for the first time) takes into account the key size of the cipher. Using this metric, we could compare block ciphers with different key sizes in a fair way. To compare RoadRunneR with existing lightweight ciphers, we used this metric and the classical ones. We gave comparison results for both the original round numbers and in the round numbers as suggested in FOAM approach. We used ATtiny45 for benchmarking, since it is one of the lowest cost 8-bit CPUs and there are many recent ciphers implemented in this device in the literature.

The organization of the rest of the paper is as follows: In Section 2, we define our new cipher RoadRunneR and give the design criteria of it. Preliminary cryptanalysis of RoadRunneR against known attacks is presented in Section 3. Our new comparison metric for block ciphers is given in Section 4. We give performance results of RoadRunneR and compare it with existing block ciphers using our new metric and other known metrics in Section 5. Section 6 concludes the paper.

\section{Definition and Design Rationale of RoadRunneR}

In the design of RoadRunneR, our main objectives were the following:

1. Implementation efficiency in 8-bit CPUs,

2. No table and SRAM usage,

3. Low decryption overhead,

4. Provable security like in wide trail design strategy [17]

We could achieve these objectives as shown in the rest of the paper. Our main focus was on reducing memory. This is because low cost 8-bit CPUs have program memory of only a few kilobytes (KB). In most applications this memory is shared by some other algorithms (such as interrupt service routines) and possibly a real time operating system. So reducing memory footprint is beneficial in our target platform.

In [39], it is stated that a hardware implementation of a lightweight block cipher targeting RFID tags and WSNs should cost less than 2000 gate equivalent (GE). For software implementations there is no stated bound, but we believe that $1 \mathrm{~KB}$ memory should not be exceeded for a lightweight block cipher implementation.

\subsection{General Structure}

RoadRunneR is a Feistel-type block cipher, shown in Figure 1, with 64-bit block size and 80-bit or 128-bit key lengths. 80-bit key requires 10 rounds and 128-bit version is 12 rounds. Initial and final round whitening is used which XOR's the 
whitening keys $\left(W K_{0}\right.$ and $\left.W K_{1}\right)$ to the left part of the state. There is no swap operation in the final round. Decryption uses the same round function where the order of whitening keys, round keys and constants are reversed.

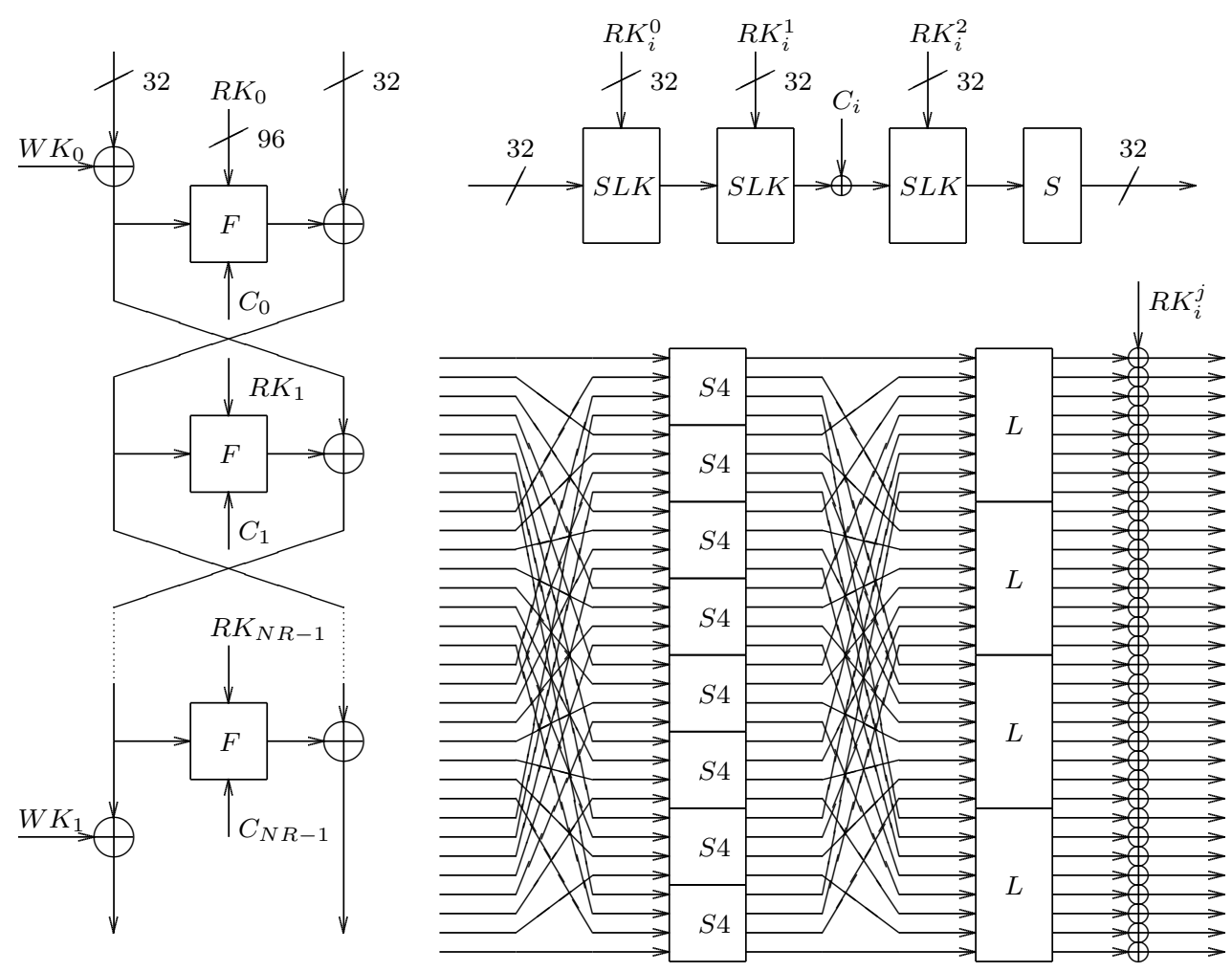

Fig. 1. Figures of functions in RoadRunneR. Feistel structure on left, $F$ function on top right, and $S L K$ function on bottom right.

If the state is shown as $x_{0}\left\|x_{1}\right\| \ldots \| x_{7}$, each round function $F$ takes most significant (leftmost) 4 bytes of the state, that is $x_{0}\left\|x_{1}\right\| x_{2} \| x_{3}$, as input data, 1-byte $C_{i}$ as constant, and 96-bit round key. Output of $F$ is XORed to $x_{4}\left\|x_{5}\right\| x_{6} \| x_{7} . F$ is a 4 round substitution-permutation-network (SPN) type function as shown on top right of Figure 1. In that figure, $S L K$ is the consecutive application of S-box layer $(S)$, diffusion layer $(L)$, and key addition $(K)$, as shown on the bottom right. The last function $S$ is the same S-box layer in $S L K$. After the second $S L K$ function, round constant is XORed to the least significant byte (rightmost byte, i.e., $\left.x_{3}\right)$ of the state. For round $i=0,1, \ldots, N R-1$, the round constant is $C_{i}=N R-i$, where $N R$ is the number of rounds, and $C_{i}$ is represented as 
8-bit little endian integer, that is $12=00001100,11=00001011$, etc. Round constants prevent simple slide attacks [8] and makes the round function to be different for different rounds. 4-round SPN-like structure ensures high number of active S-boxes for an active $F$.

In the $S L K$ function, first a bitslice S-box layer is applied on 4 words of 8 bits. This layer can be seen as parallel application of 4-bit S-boxes to the $i^{\text {th }}$ bit of each word, and writing the outputs to the same location ( $i^{\text {th }}$ bit) again. This is the best S-box in terms of security and efficiency (on CPUs) found in [42]. S-box layer is described in Section 2.2. After the S-box layer, a diffusion matrix $L$ is applied to each byte independently to ensure diffusion inside the bits of a byte. $L$ is designed such that it provides good diffusion and can be efficiently implemented even on the simplest CPUs. Definition and design criteria of $L$ will be described in Section 2.3.

\subsection{S-box layer}

Using bitslice S-boxes has become more popular in the last 10 years, especially with the recent advances in lightweight cryptography. Block ciphers such as NOEKEON[16], SEA[41], PRIDE[3], and RECTANGLE[47] use bitslice S-boxes with different S-box layer design strategies. Bitslice S-box structure has advantage in both hardware and software implementations. In software, the permutations before and after the S-boxes disappear. In hardware, on the other hand, they can be implemented by a simple wiring which consumes no extra area. Since S-boxes of large bit size and high non-linearity have a complicated circuit representation, 3-bit and 4-bit S-boxes are used in bitslice ciphers.

In RoadRunneR, an efficient bitslice S-box is used so that it can be implemented in a small number of bit-wise operations on CPU words. The table of S-box is given below:

\begin{tabular}{|c|l|l|l|l|l|l|l|l|l|l|l|l|l|l|l|l|}
\hline Input & 0 & 1 & 2 & 3 & 4 & 5 & 6 & 7 & 8 & 9 & $\mathrm{~A}$ & $\mathrm{~B}$ & $\mathrm{C}$ & $\mathrm{D}$ & $\mathrm{E}$ & $\mathrm{F}$ \\
\hline Output & 0 & 8 & 6 & $\mathrm{D}$ & 5 & $\mathrm{~F}$ & 7 & $\mathrm{C}$ & 4 & $\mathrm{E}$ & 2 & 3 & 9 & 1 & $\mathrm{~B}$ & $\mathrm{~A}$ \\
\hline
\end{tabular}

This S-box was found in [42] by a brute force search on possible assembly code combinations. The search space was restricted to 4 input words, a single temporary word, and following instructions: AND, OR, XOR, NOT , and MOV. For the best 4-bit S-boxes, maximal correlation and differential probabilities are $2^{-2}$. They experimentally found that the minimum number of instructions to generate such a bitslice S-box layer is 9. The selected S-box in RoadRunneR satisfies this property. The assembly code of the S-box for Atmel's 8-bit CPU's is as follows (X0 is the most significant byte entering the S-box layer):

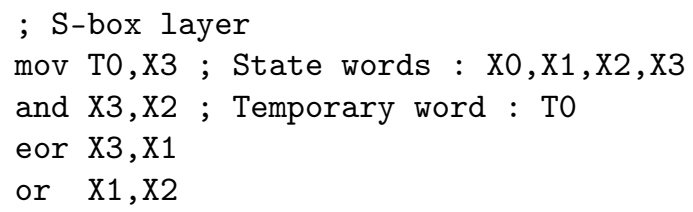


eor $\mathrm{X} 1, \mathrm{X} 0$

and $\mathrm{X} 0, \mathrm{X} 3$

eor $\mathrm{XO}, \mathrm{TO}$

and $\mathrm{T} 0, \mathrm{X} 1$

eor $\mathrm{X} 2, \mathrm{~T} 0$

\subsection{Diffusion layer}

After the bitslice S-box layer, we used a linear function on each byte of the state to provide diffusion inside 8-bit words. So we needed an efficient linear function operating on bytes. One classical solution for such a linear function for CPUs is using XOR of shifted and rotated values of the input word.

On ATtiny45 (and on most low cost 8-bit CPUs), there is no parametric shift or and no rotation instruction. So, to shift and/or rotate a byte for parametric values multiple cycles are necessary which consumes program memory and clock cycles. 1-bit left rotation can be done in 2 instructions if ADC instruction is used, whereas 1-bit right rotation can be done in 3 cycles using BST and BLD instructions. There is another instruction that swaps halves of a byte, which results in a 4-bit rotation.

Using these instructions, we try to build linear functions of the form $L(x)=$ $(x \lll i) \oplus(x \lll j) \oplus(x \lll k)$ to use in RoadRunneR, where $x \lll i$ represents $i$-bit rotation of the CPU word $x$ to the left. Linear layers of this form are guaranteed to be invertible and all have branch number 4 . Branch number of a matrix $L$ is defined as follows:

$$
B N(L)=\min _{x \neq 0}\{h w(x)+h w(L(x))\}
$$

where $h w(x)$ denotes the Hamming weight of a binary vector $x$. This number gives the minimum number of active S-boxes in two consecutive rounds. Besides the branch number, we calculated the minimum number of active S-boxes in 4 round SPN structure of $F$ with each $L$ matrix candidate. Table 1 shows the best linear functions (less then 15 instructions for two matrix multiplications) found in our search :

Table 1. Best $L$ matrices under given constraints.

\begin{tabular}{|l|c|c|c|}
\hline Matrix & $i, j, k$ & $\begin{array}{l}\text { \# of instructions (for } \\
\text { two matrix mult.) }\end{array}$ & $\begin{array}{c}\text { Minimum \# of Active } \\
\text { S-boxes in } F\end{array}$ \\
\hline$L_{1}$ & $0,1,2$ & 13 & 10 \\
\hline$L_{2}$ & $0,1,4$ & 11 & 8 \\
\hline$L_{3}$ & $0,1,5$ & 11 & 8 \\
\hline$L_{4}$ & $0,4,5$ & 11 & 8 \\
\hline$L_{5}$ & $1,4,5$ & 11 & 8 \\
\hline
\end{tabular}


From the Table 1, we have chosen $L_{1}$ as our diffusion layer matrix since it provides good diffusion and performance. The minimum number of differentially active S-boxes in an active $F$ using the above linear layers is calculated in a truncated manner, i.e., it is independent of the selected S-box. We could analyze this using an observation in [25] which gives:

$$
\alpha=x_{0} \vee x_{1} \vee x_{2} \vee x_{3} \leadsto L\left[x_{0}\right] \vee L\left[x_{1}\right] \vee L\left[x_{2}\right] \vee L\left[x_{3}\right]=\beta
$$

where $x_{i}$ 's are 8 -bit words, set bits in $\alpha$ and $\beta$ gives the active S-box positions entering into the S-box layer and after the linear layer respectively. Since there are multiple choices for $x_{i}$ 's to produce the same $\alpha$, some input truncated active S-box pattern will have multiple possible outputs, which we call transitions. $\alpha \leadsto \beta$ means that there is a transition from $\alpha$ to $\beta$. Since word size is 8 bits and there are 4 words, we could search for all possible transitions of active S-box positions while passing an $S L K$ using an exhaustive search of $2^{32}$ complexity. Here we tried all possible values of $x_{0}\left\|x_{1}\right\| x_{2} \| x_{3}$ to generate $\alpha \sim \beta$ transitions.

Using the truncated transitions, we generated a directed graph of 256 vertices. In this graph, vertices are 8-bit numbers representing active S-box positions. If vertices $\alpha$ and $\beta$ satisfy $\alpha \leadsto \beta$, this is shown as a directed edge from the vertex $\alpha$ to vertex $\beta$. Using that graph, starting from all possible vertices (except 0 ), we tried all possible directed paths of 4 vertices, summing the Hamming weights as the number of active S-boxes. Minimum weight in these paths give the minimum number of active S-boxes in an active $F$. Linear characteristics follow very similar patterns because of the definition of matrices and $F$ function's symmetric structure.

The selected matrix have single non-trivial fixed point which is FF in hexadecimal notation. In truncated active S-box transition notation, we have the following fixed points: $77,7 \mathrm{~F}, \mathrm{BB}, \mathrm{BF}, \mathrm{DD}, \mathrm{DF}, \mathrm{EE}, \mathrm{EF}, \mathrm{F} 7, \mathrm{FB}, \mathrm{FD}, \mathrm{FE}, \mathrm{FF}$. So we can say that there are at least 6 active S-boxes whenever an analysis require to use the same active S-box positions before and after $S L K$.

The AVR assembly code for 2 matrix multiplication is given below. The rationale behind using two matrix multiplication is to use single cycle 16-bit copy operation MOVW on inputs of our matrix ( $1 \mathrm{sb}$ and msb denotes least and most significant bits respectively).

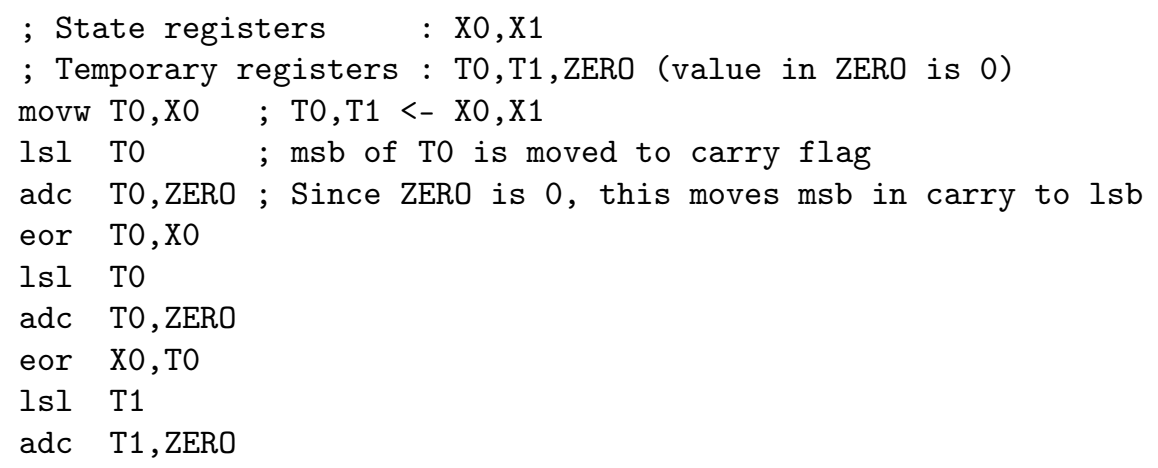


eor $\mathrm{T} 1, \mathrm{X} 1$

Isl T1

adc T1,ZERO

eor $\mathrm{X} 1, \mathrm{~T} 1$

\section{$2.4 \quad$ Key schedules}

RoadRunneR can take 80-bit or 128-bit keys. RoadRunneR-80 and RoadRunneR128 denotes 80-bit and 128-bit key versions respectively. For both key sizes, key schedules have the same simple description: Start from the beginning of the master key, whenever a new 32-bits of key material is required, continue on the master key in a circular way.

For example, if master key is 128 -bit, it will be divided into 4 words of 32-bit as $A\|B\| C \| D$. Initial whitening key is $A$, first round key is $B-C-D$, second round key is $A-B-C$, etc. Same key words appear in the same place in a period of 4 rounds. For 80-bit key schedule, master key is divided into 5 words of 16-bit : $A\|B\| C\|D\| E$. In this nomenclature, whitening key is $A \| B$; first round key is $(C \| D)-(E \| A)-(B \| C)$; second round key is $(D \| E)-(A \| B)-(C \| D)$, etc. There is a 5 -round period in this schedule, so round number is chosen as a multiple of 5 . Complete list of master key words used in rounds is given in Table 2.

Table 2. Key Schedules of RoadRunneR.

\begin{tabular}{|c|c|c|c|}
\hline \multicolumn{2}{|c|}{$\begin{array}{l}\text { 80-bit Key Schedule } \\
\text { Master key }=A\|B\| C\|D\| E\end{array}$} & \multicolumn{2}{|c|}{$\begin{array}{l}\text { 128-bit Key Schedule } \\
\text { Master key }=A\|B\| C \| D\end{array}$} \\
\hline \multicolumn{2}{|c|}{ Initial Whitening : $A \| B$} & \multicolumn{2}{|c|}{ Initial Whitening : $A$} \\
\hline Rounds & Key Words & Rounds & Key Words \\
\hline 0,5 & $(C \| D)-(E \| A)-(B \| C)$ & $0,4,8$ & $B-C-D$ \\
\hline 1,6 & $(D \| E)-(A \| B)-(C \| D)$ & $1,5,9$ & $A-B-C$ \\
\hline 2,7 & $(E \| A)-(B \| C)-(D \| E)$ & $2,6,10$ & $D-A-B$ \\
\hline 3,8 & $(A \| B)-(C \| D)-(E \| A)$ & $3,7,11$ & $C-D-A$ \\
\hline 4,9 & $(B \| C)-(D \| E)-(A \| B)$ & & \\
\hline \multicolumn{2}{|c|}{ Final Whitening : $C \| D$} & \multicolumn{2}{|c|}{ Final Whitening : $B$} \\
\hline
\end{tabular}

\section{Security of RoadRunneR}

For the attacks on reduced round numbers, we assume that there is an initial and final whitening in reduced versions on the left hand side of the cipher, and no swap is applied in the last round. Final whitening is the next key word in the key schedule.

Whitening is only applied to left sides to prevent attackers from using known bits of the cipher's intermediate round values. There is no need to XOR whitening 
key on right halves to provide this property. In most of the attacks, extending the attack by even one round is not possible since this requires to search for all 128-bit keys (in 128-bit key version) because of the whitening. So, we can say that whitening keys play a crucial security role in RoadRunneR, and cannot be omitted in the attacks.

Since the key schedule uses the master key without any change while using in the rounds, we have no security claim against related key attacks. Related key attacks are easier to defend on the protocol level, and some of the lightweight ciphers do not consider this attack as well, such as PRINCE and PRIDE. Hence we used this assumption in RoadRunneR. In fact, each $F$ can be passed with only two active S-boxes in a related key attack, with total of 24 active S-boxes. This total number may be further reduced in a more detailed analysis.

\subsection{Differential and linear attacks}

Differential attack [6] and linear attack [37] are two most successful attacks on block ciphers. RoadRunneR has provable bounds on minimum number of differentially active S-boxes, which shows that there is no useful differential characteristic for 5 rounds or more. Since the transpose of our diffusion layer have similar properties and $F$ function have a symmetric structure, differential and linear properties (in a truncated manner) of RoadRunneR are the same. So whenever we mention active S-boxes, we mean both differentially and linearly active S-boxes.

As we have mentioned in Section 2.3, the minimum number of active S-boxes in an active $F$ is 10 . Note that this number is better than the value suggested by branch number, which is 8 for 4 rounds (since branch number is 4 ). There are 8 truncated trails of 10 and 11 active S-boxes, and all of them start with 1 active Sboxes. Moreover, the characteristics starting with the same input pattern follow the same path for round 2 and 3, that is only the last round is different for minimal weight paths. These paths are given below.

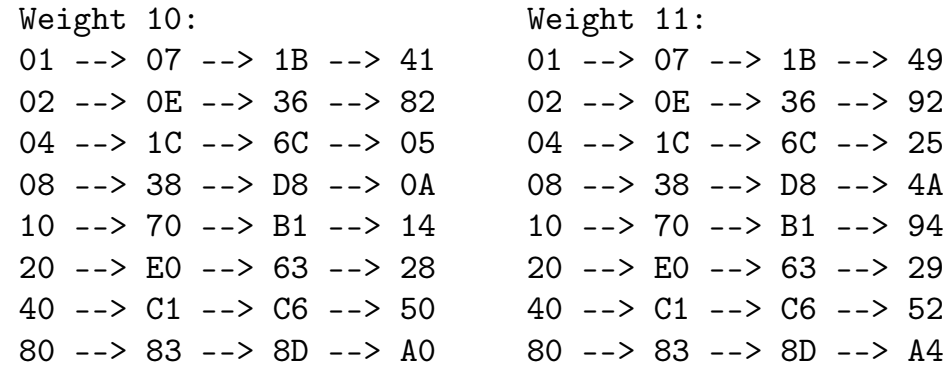

We experimentally checked some high probability differential characteristics of $F$ starting with one active S-box to see if the probability of characteristics and differentials are close or not. In our experiments, we did not see any significant increase in differential probability, from the theoretically calculated characteristic probability. So we assumed that each active S-box multiplies the probability with $2^{-2}$, and an active $F$ has approximately $2^{-20}$ probability. 
We also calculated the minimum number of active S-boxes in $r$-round RoadRunneR for $4 \leq r \leq 6$, again in a truncated manner. This is done by an exhaustive search, thanks to the graph we mentioned in Section 2.3. Utilizing that graph, we could generate all possible truncated active S-box transitions on $F$ function, together with their minimum number of active S-boxes. In our search of minimum number of active S-boxes in $r$-rounds RoadRunneR, we try all possible truncated input difference patterns to the cipher and follow an $r$-round path using branching over $F$ and Feistel XORs, counting number of active S-boxes in all $F$ functions. Whenever two truncated difference meet in an XOR of Feistel scheme, we tried both cases with a difference and without difference. Table 3 shows minimum number of active S-boxes for rounds from 4 up to 6, together with the percentage of active S-boxes.

Table 3. Minimum number of truncated active S-boxes for rounds.

\begin{tabular}{|l|l|l|l|}
\hline Round & 4 & 5 & 6 \\
\hline \# of Active S-boxes & 26 & 36 & 48 \\
\hline Percentage & $20.3 \%$ & $22.5 \%$ & $25 \%$ \\
\hline
\end{tabular}

Note that these bounds are better than the classical bounds on Feistel ciphers with invertible $F$ function, which gives 2, 3 and 4 active $F$ functions in 4, 5, and 6 consecutive rounds respectively. In that classical approach, since an active $F$ has at least 10 active S-boxes, the bound is 20,30 and 40 active S-boxes for 4, 5 , and 6 rounds, whereas we found 26, 36, and 48 active S-boxes for these round numbers in our search. We believe that the active S-box percentage values are very good for such a lightweight linear layer. Table 3 proves that there is no useful differential characteristic (or linear trail) in 5 or more rounds of RoadRunneR, since the probability is at least $2^{-72}$.

We listed all paths with minimum number of S-boxes in our search. By observing the trails, we saw that there were no clustering in best trails, i.e. no paths starting and ending with the same active S-box positions in 5 rounds. This gives confidence that characteristic and differential probabilities are very close in the whole cipher. Hence, we believe that 5 round RoadRunneR is secure against classical differential and linear attacks.

There are many attacks derived from differential attack and linear attack. Some examples are: higher order differential attack [34], boomerang attack [43], multidimensional linear attack [13], differential-linear attack [35], etc. In general, these extension attacks do not give better results then classical differential and linear attacks. We think that the same is true for RoadRunneR. Since all key material is used in the first and last rounds with the use of whitening keys, it is hard to apply $1 \mathrm{R}$ and $2 \mathrm{R}$ attacks. 


\subsection{Impossible differential attack}

In impossible differential attack [7], truncated differentials with probability 1 are used to find a difference contradiction in middle rounds. This contradiction is then used to eliminate wrong keys in the extra rounds added before and after the characteristic. For Feistel ciphers, there is a generic 5 round impossible differential characteristic [31] as follows:

$$
(0, \Delta \alpha) \stackrel{5}{\rightarrow}(0, \Delta \alpha)
$$

Since the round function of RoadRunneR has a four round SPN structure, we could not find impossible differential characteristic for more than 5 rounds. On the other hand, all key material is used in the first and last round when whitening keys are considered, so we believe that impossible differential attack cannot be applied to more than 6 rounds of RoadRunneR.

\subsection{Integral attack}

The integral attack (or square attack) was demonstrated in [14] as a custom attack to SQUARE block cipher. It was also applied to Rijndael which become AES, and many other ciphers. In this attack, all possible values are given to specific bit blocks in the plaintext, and other bits are kept constant. After some rounds of encryption, fixed sum (generally zero) in specific ciphertext bit locations are expected.

Because of the 4 round SPN structure in $F$ function, giving all possible values to a single S-box do not give too many rounds in an integral attack. The best attack can be achieved by 32-bit active block on the right half of the plaintexts as in the following:

$$
(0, A) \rightarrow(A, 0) \rightarrow(A, A) \rightarrow(B, A) \rightarrow(?, B)
$$

Here, $A$ denotes an active 32 -bit block where all possible values are seen. $B$ is a balanced block, that is XOR sum of values are zero. An undetermined block is represented by a ? mark. This characteristic cannot be extended to more rounds. Therefore, we do not think that square attack threatens RoadRunneR for more than 6 rounds.

\subsection{MITM-type attacks}

All state bits are affected by all key bits after 3 rounds of RoadRunneR encryption. Moreover, when the matching variable in a Meet-In-The-Middle (MITM) attack is selected in the right half of the state at the output of round 3 , it is not possible to add even 2 rounds because of the fact that all key bits affect that variable in the decryption direction after 2 rounds. Same ideas apply for 2 rounds at the beginning and 3 rounds at the end case due to the Feistel structure. Hence, MITM attack cannot be applied to more than 4 rounds. 
There are some extensions of MITM attack such as multidimensional MITM [48], Demirci-Selçuk attack [18], and MITM attacks with tabulation and differential enumeration techniques [22]. These attacks generally uses truncated differential characteristics with high probability over multiple rounds. In the case of RoadRunneR, since round function $F$ is a 4-round SPN, we believe that these attacks are not more effective than basic MITM attack.

\subsection{Side-Channel Attacks}

Lightweight ciphers are vulnerable to side-channel attacks. The attacker can access low cost devices that have the secret key, and can measure encryption time, power dissipation, radiation, etc. Therefore, mechanisms to protect the cipher against such attacks may be necessary in some applications.

It is shown in [15] that ciphers with bitslice nonlinear layer are easier to defend against side-channel attacks such as differential power analysis (DPA) [33]. Since RoadRunneR has a bitslice non-linear layer, we can say that the additional overhead caused by DPA protecting mechanisms is low for our cipher.

\section{A New Efficiency Metric For Block Ciphers : $S T / A$}

In this section, we propose a new metric called $S T / A$, which we read as $S e c u$ rity times Throughput over Area. In this new metric, we insert key size to the efficiency metric formulae since there is no fair way to compare block ciphers of different key length in the literature. We extend $\frac{\text { Throughput }}{\text { Area }}$ metric by multiplying it with the key size, so we have:

$$
S T / A=\frac{\text { KeySize } \times \text { Throughput }}{\text { Area }}
$$

where KeySize is the bit size of key used in the cipher, Throughput is given in bit-per-second, and Area is gate equivalent (GE) in hardware or memory usage in software.

We inserted the key size by multiplication, hence increase in the key size increases the efficiency of a cipher. Moreover, other parameters affect the metric in a multiplicative manner. So another mathematical operation, such as addition, would have less meaning. In our metric, algorithms with equal round function and round number for different key sizes, such as PRESENT, will have better efficiency in higher key size. On the other hand, existing metrics in the literature do not differentiate these key sizes. This is an other evident that our metric makes more fair comparison even in this specific case.

In [20], the metric is calculated in an additive manner. On the other hand, all previous methods and $S T / A$ are multiplicative, that is all performance value are multiplied. We think that multiplying is a more useful technique. For example, let $E_{1}$ and $E_{2}$ be two ciphers which will be compared. Also assume that all performance indicators are the same for both cipher except area values, where $E_{1}$ has area $A$ and $E_{2}$ has area $2 \times A$. By the multiplication method, we can say 
that $E_{1}$ is two times better than $E_{2}$. In the summation case, however, even if the weights are equal, we would not have this ratio in efficiency values. Therefore, we multiply each indicator as in the classical $\frac{\text { Throughput }}{\text { Area }}$ formula (here throughput is multiplied by $\frac{1}{\text { Area }}$ ) in $S T / A$.

We believe that the throughput should be defined as in FOAM, but we leave this to the user of this metric. Weighting approach as in [20] can be used in $S T / A$ by taking weights as powers of area, speed and key size values, since we use a multiplicative approach. Again, this is left to the user, and we use all powers as 1.

In Section 5, we use our metric for the comparison of the efficiency of some lightweight block ciphers and our new design RoadRunneR.

\section{Performance Analysis}

We have simulated RoadRunneR for ATtiny45 processor using AVR assembly language in Atmel Studio 6.2. Implementations are encryption only, where master key and plaintext are read from SRAM, plaintext is encrypted, and written back to the same place again. There is no SRAM usage besides master key and plaintext, so only code size is given as the area performance. Loading of key and plaintext, and storing back ciphertext is included in the single block encryption time. Various optimization methods are applied. In Table 4, we give the performance result of RoadRunneR block cipher.

Table 4. Performance values of RoadRunneR- 80 and RoadRunneR-128 for different optimization methods. Compact-1 and Compact-2 are in between methods, shown as signs of possible trade-offs.

\begin{tabular}{|l|c|c|c|}
\hline Key Size & Optimization & Code Size (Byte) & Time (cycle) \\
\hline \hline 80 & Area & 202 & 3279 \\
\hline 80 & Speed & 386 & 2091 \\
\hline \hline 128 & Area & 196 & 3819 \\
\hline 128 & Compact-1 & 228 & 2461 \\
\hline 128 & Compact-2 & 402 & 2171 \\
\hline 128 & Speed & 502 & 2025 \\
\hline
\end{tabular}

Area optimized 80-bit key version has slightly more area than 128-bit key version. This is because of the more complex key schedule in 80-bit key. Optimization column in Table 4 shows the various implementation methods we apply. Area optimization method gives the smallest code size we could achieve. This is done by extensive use of subroutines which saves program memory. Speed optimization, on the other hand, use no subroutines to avoid extra cycles required by branching to subroutines. Compact methods are described below: 
- Compact-1 : This is derived from area optimized version. Some subroutines are removed and repeating codes are written for them.

- Compact-2 : Derived from speed optimized version. Key selection part in speed optimized version changed to a subroutine.

There are more trade-offs with different code size/clock cycle properties but we did not include them in the paper. From this and Table 4, we see that RoadRunneR have good throughput/area/security trade-off properties. If we start from the speed optimized version, we can reduce the area more than half and pay a speed penalty of less than half. Fastest implementation is still relatively small, and the smallest implementation is not that slow.

Comparison of RoadRunneR with some other ciphers is given in Table 5. We show four comparison metric values for each cipher. Metrics are explained below:

- $T / A$ is the classical $\frac{\text { Throughput }}{\text { Area }}$ metric.

- $T / A$-FOAM is the same metric with the throughput definition in FOAM.

- $S T / A$ is calculated by multiplying the $T / A$ by the key size.

- $S T / A$-FOAM is calculated by multiplying the $T / A$-FOAM by the key size.

Instead of using $\frac{\text { Throughput }}{\text { Area }}$, we chose to use Area $\times$ Time product (time to produce 1 byte, i.e., Cycle/Byte) as in the comparisons in [28], which gives the same order. Here, in contrast to $\frac{\text { Throughput }}{\text { Area }}$, small values are better. We also normalize each comparison metric column for better understanding. For the normalization, we divide all numbers in the column with the smallest value in that column.

In the calculation of FOAM values, we searched for the best attack on each cipher in terms of round number, and used that as round number to calculate encryption time. This calculation done by multiplying the original encryption clock count by $N R^{*} / N R$, where $N R$ is the original round number and $N R^{*}$ is the round numbers calculated by the above idea. We do not exclude any initial setup since we do not know each implementation in detail. We also excluded related-key attacks since we have no security claim for this type of attack. For NOEKEON and SEA, we use the bounds found by the designers because of the lack of cryptanalysis in the literature on these ciphers.

In Table 5, (A) and (S) stands for area optimized and speed optimized implementations, respectively. (C1) and (C2) are compact implementations as defined previously. We write RRR as an abbreviation of RoadRunneR. We did not include SERPENT-128 and CLEFIA-128 in the list since they were far behind any of the other ciphers in the table in terms of efficiency metrics.

Table 5 shows that, the best cipher in terms of our metrics and classical metrics is SPECK family. But this family follows the Addition-Rotation-XOR (ARX) design principle and lacks the provable security properties. So, the round number selection of SPECK have no scientific rationale. Moreover, in an attack paper on Simon [38], authors claim that truncated differential characteristics to be found in the future may extend their 26 round attack to more rounds on the cipher. Therefore, if we exclude SIMON and SPECK, we have the following picture among remaining implementations: 
Table 5. Comparison of some block ciphers implemented on ATtiny45. RRR stands for RoadRunneR, (A), (S), (C1), and (C2) are implementations methods. Comparison metrics are normalized where small values are better.

\begin{tabular}{|c|c|c|c|c|c|c|c|c|c|c|}
\hline Cipher & $\begin{array}{r}\text { Block } \\
\text { size }\end{array}$ & $\begin{array}{r}\text { Key } \\
\text { size }\end{array}$ & $\begin{array}{l}\text { Attacked } \\
\text { Rounds }\end{array}$ & $\begin{array}{l}\text { Mem. } \\
\text { [byte] }\end{array}$ & $\begin{array}{l}\text { Enc. } \\
\text { Clks }\end{array}$ & $\begin{array}{r}\text { Cyc./ } \\
\text { Byte }\end{array}$ & $T / A$ & $\begin{array}{r}T / A \\
\text { FOAM }\end{array}$ & $S T / A$ & $\begin{array}{r}S T / A \\
\text { FOAM }\end{array}$ \\
\hline AES [24] & 128 & 128 & 7/10 [19] & 1570 & 3159 & 1971 & 11.83 & 12.35 & 11.11 & 12.38 \\
\hline PRESENT [24] & 64 & 128 & $26 / 31[9]$ & 660 & 10792 & $1349:$ & 33.97 & 42.51 & 31.91 & 42.58 \\
\hline SEA [3] & 96 & 96 & 72/93 [41] & 386 & 17745 & 1479 & 21.78 & 25.43 & 27.26 & 33.96 \\
\hline NOEKEON [23] & 128 & 128 & 9/12 [16] & 364 & 23517 & 1470 & 20.41 & 22.84 & 19.17 & 22.88 \\
\hline PRINCE [3] & 64 & 128 & $12 / 12[27]$ & 1108 & 3614 & 451 & 19.01 & 28.49 & 19.94 & 28.54 \\
\hline ITUBEe [28] & 80 & 80 & $\mathbf{1 0} / \mathbf{2 0}[40]$ & 716 & 2607 & 261 & 7.13 & 5.32 & 10.72 & 8.53 \\
\hline PRIDE [3] & 64 & 128 & $19 / 20[46]$ & 266 & 1514 & 189 & 1.92 & 2.72 & 1.80 & 2.73 \\
\hline RRR-80 (A) & 64 & 80 & $6 / 10$ & 202 & 3279 & 410 & 3.16 & 2.83 & 4.75 & 4.53 \\
\hline RRR-80 (S) & 64 & 80 & $6 / 10$ & 386 & 2091 & 261 & 3.85 & 3.45 & 5.78 & 5.53 \\
\hline $8(\mathrm{~A})$ & 64 & 128 & $6 / 12$ & 196 & 3819 & 477 & 3.57 & 2.66 & 3.35 & 2.66 \\
\hline RRR-128 (C1) & 64 & 128 & $6 / 12$ & 228 & 2461 & 308 & 2.68 & 2.00 & 2.51 & 2.00 \\
\hline RRR-128 (C2) & 64 & 128 & $6 / 12$ & 402 & 2171 & 271 & 4.16 & 3.10 & 3.91 & 3.11 \\
\hline RRR-128 (S) & 64 & 128 & $6 / 12$ & 502 & 2025 & 253 & 4.85 & 3.62 & 4.55 & 3.62 \\
\hline SIMON [5] & 64 & 128 & $26 / 42[2]$ & 282 & 2000 & 250 & 2.69 & 2.37 & 2.53 & 2.37 \\
\hline SPECK [5] & 64 & 96 & $18 / 26[21]$ & 182 & 1152 & 144 & 1.00 & 1.03 & 1.28 & 1.38 \\
\hline SPECK [5] & 64 & 128 & $17 / 27[21]$ & 186 & 1200 & 150 & 1.06 & 1.00 & 1.00 & 1.00 \\
\hline
\end{tabular}

RoadRunneR is the best algorithm in terms of code size (except speed optimized and $\mathrm{C} 2$ implementations) and security margin. When FOAM approach is not considered, i.e., in $T / A$ and $S T / A$ metrics, PRIDE outperforms all others, RoadRunneR implementations follow PRIDE. When we take into account security margins, $T / A$-FOAM and $S T / A$-FOAM metrics show that $(\mathrm{A})$ and $(\mathrm{C} 1)$ implementations have the highest rank, PRIDE and other implementations of RoadRunneR follow them. Throughput of RoadRunneR is not the best in any implementation but the fastest implementation of it has the rank 3 among 8 ciphers. We think that RoadRunneR is fast enough for most applications with low cost 8-bit CPUs. Bold numbers show the best values in their column except SIMON and SPECK family. Multiple values in RoadRunneR implementations are written bold if they are better then all previous results.

\section{Conclusion and Future Work}

A very efficient Feistel type bitslice block cipher, RoadRunneR, with 64-bit block size and 80-bit or 128-bit key length is presented. RoadRunneR is a perfect choice for devices with very restricted memory resources and for applications requiring reasonable throughput expectations. Our cipher has a high security margin in contrast to most of other lightweight block ciphers. We simulated 
RoadRunneR on ATtiny45 devices by using Atmel Studio 6.2, for which there are implementation results of recent lightweight ciphers in the literature.

To compare our cipher and other ciphers with different key lengths, we proposed a new comparison metric which considers throughput, area and key size. When two ciphers of similar area and throughput values are achieved, the one with larger key size will have a higher rank in this metric. Our metric is the first one to use key length in the literature.

Implementation results show that RoadRunneR is a competitive candidate in all metrics in the literature and in our new metric. In our comparisons, only SPECK and PRIDE performed better than RoadRunneR in some metrics, but SPECK lacks a security proof and there is a 19 out of 20 round differential attack on PRIDE. In this sense, we think that RoadRunneR is a good alternative to current lightweight block ciphers.

Future Work: Methods for counting minimum number of active S-boxes in an $r$-round $(r>2)$ bitslice SPN cipher (like PRIDE and RECTANGLE) for larger than 8-bit word size is a challenge. If an efficient method can be found, this may be used to generate and evaluate binary matrices used in bitslice ciphers, together with their efficiency. Moreover, general frameworks for determining power weights for area, throughput, and key size (security) in $S T / A$ for various implementation platforms is necessary. In the current state, we take all powers as 1 , but some implementations may require very constrained area or time characteristics. How to find most useful powers is an open problem. We also leave efficient hardware implementations of RoadRunneR as a future work.

\section{References}

1. ATtiny45. http://www.atmel.com/devices/attiny45.aspx, Visited on June 18, 2015.

2. Farzaneh Abed, Eik List, Stefan Lucks, and Jakob Wenzel. Differential and linear cryptanalysis of reduced-round simon. Technical report, Citeseer, 2013.

3. Martin R. Albrecht, Benedikt Driessen, Elif Bilge Kavun, Gregor Leander, Christof Paar, and Tolga Yalçin. Block ciphers - focus on the linear layer (feat. PRIDE). In Juan A. Garay and Rosario Gennaro, editors, Advances in Cryptology - CRYPTO 2014 - 34th Annual Cryptology Conference, Santa Barbara, CA, USA, August 1721, 2014, Proceedings, Part I, volume 8616 of Lecture Notes in Computer Science, pages 57-76. Springer, 2014.

4. Stéphane Badel, Nilay Dagtekin, Jorge Nakahara Jr., Khaled Ouafi, Nicolas Reffé, Pouyan Sepehrdad, Petr Susil, and Serge Vaudenay. ARMADILLO: A multipurpose cryptographic primitive dedicated to hardware. In Mangard and Standaert [36], pages 398-412.

5. Ray Beaulieu, Douglas Shors, Jason Smith, Stefan Treatman-Clark, Bryan Weeks, and Louis Wingers. The simon and speck families of lightweight block ciphers. Cryptology ePrint Archive, Report 2013/404, 2013. http://eprint.iacr.org/.

6. Eli Biham and Adi Shamir. Differential Cryptanalysis of DES-like Cryptosystems. In Alfred Menezes and Scott A. Vanstone, editors, CRYPTO, volume 537 of Lecture Notes in Computer Science, pages 2-21. Springer, 1990. 
7. Alex Biryukov. Impossible differential attack. In Henk C. A. van Tilborg, editor, Encyclopedia of Cryptography and Security. Springer, 2005.

8. Alex Biryukov and David Wagner. Slide attacks. In Knudsen [30], pages 245-259.

9. Céline Blondeau and Kaisa Nyberg. Links between truncated differential and multidimensional linear properties of block ciphers and underlying attack complexities. In Phong Q. Nguyen and Elisabeth Oswald, editors, Advances in Cryptology - EUROCRYPT 2014 - 33rd Annual International Conference on the Theory and Applications of Cryptographic Techniques, Copenhagen, Denmark, May 11-15, 2014. Proceedings, volume 8441 of Lecture Notes in Computer Science, pages 165-182. Springer, 2014.

10. Andrey Bogdanov, Lars R. Knudsen, Gregor Leander, Christof Paar, Axel Poschmann, Matthew J. B. Robshaw, Yannick Seurin, and C. Vikkelsoe. PRESENT: An Ultra-Lightweight Block Cipher. In Pascal Paillier and Ingrid Verbauwhede, editors, CHES, volume 4727 of Lecture Notes in Computer Science, pages 450-466. Springer, 2007.

11. Andrey Bogdanov, Gregor Leander, Christof Paar, Axel Poschmann, Matthew J. B. Robshaw, and Yannick Seurin. Hash functions and RFID tags: Mind the gap. In Elisabeth Oswald and Pankaj Rohatgi, editors, Cryptographic Hardware and Embedded Systems - CHES 2008, 10th International Workshop, Washington, D.C., USA, August 10-13, 2008. Proceedings, volume 5154 of Lecture Notes in Computer Science, pages 283-299. Springer, 2008.

12. Julia Borghoff, Anne Canteaut, Tim Güneysu, Elif Bilge Kavun, Miroslav Knezevic, Lars R. Knudsen, Gregor Leander, Ventzislav Nikov, Christof Paar, Christian Rechberger, Peter Rombouts, Søren S. Thomsen, and Tolga Yalçin. PRINCE A Low-Latency Block Cipher for Pervasive Computing Applications - Extended Abstract. In Xiaoyun Wang and Kazue Sako, editors, ASIACRYPT, volume 7658 of Lecture Notes in Computer Science, pages 208-225. Springer, 2012.

13. Joo Yeon Cho, Miia Hermelin, and Kaisa Nyberg. A new technique for multidimensional linear cryptanalysis with applications on reduced round serpent. In Pil Joong Lee and Jung Hee Cheon, editors, Information Security and Cryptology - ICISC 2008, 11th International Conference, Seoul, Korea, December 3-5, 2008, Revised Selected Papers, volume 5461 of Lecture Notes in Computer Science, pages 383-398. Springer, 2008.

14. Joan Daemen, Lars R. Knudsen, and Vincent Rijmen. The block cipher square. In Eli Biham, editor, Fast Software Encryption, 4th International Workshop, FSE '97, Haifa, Israel, January 20-22, 1997, Proceedings, volume 1267 of Lecture Notes in Computer Science, pages 149-165. Springer, 1997.

15. Joan Daemen, Michaël Peeters, and Gilles Van Assche. Bitslice ciphers and power analysis attacks. In Bruce Schneier, editor, Fast Software Encryption, 7th International Workshop, FSE 2000, New York, NY, USA, April 10-12, 2000, Proceedings, volume 1978 of Lecture Notes in Computer Science, pages 134-149. Springer, 2000.

16. Joan Daemen, Michaël Peeters, Gilles Van Assche, and Vincent Rijmen. Nessie proposal: Noekeon. 2000.

17. Joan Daemen and Vincent Rijmen. The wide trail design strategy. In Bahram Honary, editor, Cryptography and Coding, 8th IMA International Conference, Cirencester, UK, December 17-19, 2001, Proceedings, volume 2260 of Lecture Notes in Computer Science, pages 222-238. Springer, 2001.

18. Hüseyin Demirci and Ali Aydin Selçuk. A meet-in-the-middle attack on 8-round AES. In Kaisa Nyberg, editor, Fast Software Encryption, 15th International Workshop, FSE 2008, Lausanne, Switzerland, February 10-13, 2008, Revised Se- 
lected Papers, volume 5086 of Lecture Notes in Computer Science, pages 116-126. Springer, 2008.

19. Patrick Derbez and Pierre-Alain Fouque. Exhausting demirci-selçuk meet-in-themiddle attacks against reduced-round AES. In Shiho Moriai, editor, Fast Software Encryption - 20th International Workshop, FSE 2013, Singapore, March 11-13, 2013. Revised Selected Papers, volume 8424 of Lecture Notes in Computer Science, pages 541-560. Springer, 2013.

20. Daniel Dinu, Yann Le Corre, Dmitry Khovratovich, Léo Perrin, Johann Großschädl, and Alex Biryukov. Triathlon of lightweight block ciphers for the internet of things. IACR Cryptology ePrint Archive, 2015:209, 2015.

21. Itai Dinur. Improved differential cryptanalysis of round-reduced speck. Cryptology ePrint Archive, Report 2014/320, 2014. http://eprint.iacr.org/.

22. Orr Dunkelman, Nathan Keller, and Adi Shamir. Improved single-key attacks on 8-round AES-192 and AES-256. J. Cryptology, 28(3):397-422, 2015.

23. Thomas Eisenbarth, Zheng Gong, Tim Güneysu, Stefan Heyse, Sebastiaan Indesteege, Stéphanie Kerckhof, François Koeune, Tomislav Nad, Thomas Plos, Francesco Regazzoni, François-Xavier Standaert, and Loïc van Oldeneel tot Oldenzeel. Compact implementation and performance evaluation of block ciphers in attiny devices. In Aikaterini Mitrokotsa and Serge Vaudenay, editors, Progress in Cryptology - AFRICACRYPT 2012 - 5th International Conference on Cryptology in Africa, Ifrance, Morocco, July 10-12, 2012. Proceedings, volume 7374 of Lecture Notes in Computer Science, pages 172-187. Springer, 2012.

24. Susanne Engels, Elif Bilge Kavun, Christof Paar, Tolga Yalçin, and Hristina Mihajloska. A non-linear/linear instruction set extension for lightweight ciphers. In Alberto Nannarelli, Peter-Michael Seidel, and Ping Tak Peter Tang, editors, 21st IEEE Symposium on Computer Arithmetic, ARITH 2013, Austin, TX, USA, April 7-10, 2013, pages 67-75. IEEE Computer Society, 2013.

25. Vincent Grosso, Gaëtan Leurent, François-Xavier Standaert, and Kerem Varici. Ls-designs: Bitslice encryption for efficient masked software implementations. In Carlos Cid and Christian Rechberger, editors, Fast Software Encryption - 21st International Workshop, FSE 2014, London, UK, March 3-5, 2014. Revised Selected Papers, volume 8540 of Lecture Notes in Computer Science, pages 18-37. Springer, 2014.

26. Jian Guo, Thomas Peyrin, Axel Poschmann, and Matthew J. B. Robshaw. The LED Block Cipher. In Bart Preneel and Tsuyoshi Takagi, editors, CHES, volume 6917 of Lecture Notes in Computer Science, pages 326-341. Springer, 2011.

27. Jeremy Jean, Ivica Nikolic, Thomas Peyrin, Lei Wang, and Shuang Wu. Security analysis of prince. Cryptology ePrint Archive, Report 2015/372, 2015. http: //eprint.iacr.org/.

28. Ferhat Karakoç, Hüseyin Demirci, and A. Emre Harmanci. Itubee: A software oriented lightweight block cipher. In Gildas Avoine and Orhun Kara, editors, Lightweight Cryptography for Security and Privacy - Second International Workshop, LightSec 2013, Gebze, Turkey, May 6-7, 2013, Revised Selected Papers, volume 8162 of Lecture Notes in Computer Science, pages 16-27. Springer, 2013.

29. Khoongming Khoo, Thomas Peyrin, Axel Poschmann, and Huihui Yap. FOAM: searching for hardware-optimal SPN structures and components with a fair comparison. IACR Cryptology ePrint Archive, 2014:530, 2014.

30. Lars R. Knudsen, editor. Fast Software Encryption, 6th International Workshop, FSE '99, Rome, Italy, March 24-26, 1999, Proceedings, volume 1636 of Lecture Notes in Computer Science. Springer, 1999. 
31. Lars R. Knudsen. The Security of Feistel Ciphers with Six Rounds or Less. J. Cryptology, 15(3):207-222, 2002.

32. Lars R. Knudsen, Gregor Leander, Axel Poschmann, and Matthew J. B. Robshaw. PRINTcipher: A Block Cipher for IC-Printing. In Mangard and Standaert [36], pages $16-32$.

33. Paul C. Kocher, Joshua Jaffe, and Benjamin Jun. Differential power analysis. In Michael J. Wiener, editor, Advances in Cryptology - CRYPTO '99, 19th Annual International Cryptology Conference, Santa Barbara, California, USA, August 1519, 1999, Proceedings, volume 1666 of Lecture Notes in Computer Science, pages 388-397. Springer, 1999.

34. Xuejia Lai. Higher order derivatives and differential cryptanalysis. In RichardE. Blahut, Jr. Costello, DanielJ., Ueli Maurer, and Thomas Mittelholzer, editors, Communications and Cryptography, volume 276 of The Springer International Series in Engineering and Computer Science, pages 227-233. Springer US, 1994.

35. Susan K. Langford and Martin E. Hellman. Differential-linear cryptanalysis. In Yvo Desmedt, editor, CRYPTO, volume 839 of Lecture Notes in Computer Science, pages 17-25. Springer, 1994.

36. Stefan Mangard and François-Xavier Standaert, editors. Cryptographic Hardware and Embedded Systems, CHES 2010, 12th International Workshop, Santa Barbara, CA, USA, August 17-20, 2010. Proceedings, volume 6225 of Lecture Notes in Computer Science. Springer, 2010.

37. Mitsuru Matsui. Linear Cryptoanalysis Method for DES Cipher. In Tor Helleseth, editor, EUROCRYPT, volume 765 of Lecture Notes in Computer Science, pages 386-397. Springer, 1993.

38. Theodosis Mourouzis, Guangyan Song, Nicolas Courtois, and Michalis Christofii. Advanced differential cryptanalysis of reduced-round simon64/128 using largeround statistical distinguishers. Cryptology ePrint Archive, Report 2015/481, 2015. http://eprint.iacr.org/.

39. Markku-Juhani O. Saarinen and Daniel W. Engels. A do-it-all-cipher for RFID: design requirements (extended abstract). IACR Cryptology ePrint Archive, 2012:317, 2012.

40. Hadi Soleimany. Self-similarity cryptanalysis of the block cipher itubee. IET Information Security, 9(3):179-184, 2014.

41. François-Xavier Standaert, Gilles Piret, Neil Gershenfeld, and Jean-Jacques Quisquater. SEA: A scalable encryption algorithm for small embedded applications. In Josep Domingo-Ferrer, Joachim Posegga, and Daniel Schreckling, editors, Smart Card Research and Advanced Applications, 7th IFIP WG 8.8/11.2 International Conference, CARDIS 2006, Tarragona, Spain, April 19-21, 2006, Proceedings, volume 3928 of Lecture Notes in Computer Science, pages 222-236. Springer, 2006.

42. Markus Ullrich, Christophe De Canniere, Sebastiaan Indesteege, Özgül Küçük, Nicky Mouha, and Bart Preneel. Finding optimal bitsliced implementations of $4 \times$ 4-bit s-boxes. In SKEW 2011 Symmetric Key Encryption Workshop, Copenhagen, Denmark, pages 16-17, 2011.

43. David Wagner. The boomerang attack. In Knudsen [30], pages 156-170.

44. David J. Wheeler and Roger M. Needham. TEA, a Tiny Encryption Algorithm. In Bart Preneel, editor, FSE, volume 1008 of Lecture Notes in Computer Science, pages 363-366. Springer, 1994.

45. Wenling $\mathrm{Wu}$ and Lei Zhang. LBlock: A Lightweight Block Cipher. In Javier Lopez and Gene Tsudik, editors, ACNS, volume 6715 of Lecture Notes in Computer Science, pages 327-344, 2011. 
46. Qianqian Yang, Lei Hu, Siwei Sun, Kexin Qiao, Ling Song, Jinyong Shan, and Xiaoshuang Ma. Improved differential analysis of block cipher PRIDE. In Javier Lopez and Yongdong Wu, editors, Information Security Practice and Experience - 11th International Conference, ISPEC 2015, Beijing, China, May 5-8, 2015. Proceedings, volume 9065 of Lecture Notes in Computer Science, pages 209-219. Springer, 2015.

47. Wentao Zhang, Zhenzhen Bao, Dongdai Lin, Vincent Rijmen, Bohan Yang, and Ingrid Verbauwhede. RECTANGLE: A bit-slice ultra-lightweight block cipher suitable for multiple platforms. IACR Cryptology ePrint Archive, 2014:84, 2014.

48. Bo Zhu and Guang Gong. Multidimensional meet-in-the-middle attack and its applications to KATAN32/48/64. Cryptography and Communications, 6(4):313$333,2014$.

\section{A Test Vectors for 80-bit Key Length}

\begin{tabular}{|c|c|c|}
\hline laintext & Key & Cip \\
\hline 000 0000_0000 & $0000 \_0000$ & $7 \mathrm{FOB}$ \\
\hline $0000 \_0000$ _ & 8000 & 4FA2 \\
\hline EDC_BA98_7654_3 & 123_4567_89AB_CDEF_0123 & 328C_798A_0EB2_5A3B \\
\hline
\end{tabular}

\section{B Test Vectors for 128-bit Key Length}

\begin{tabular}{|c|c|c|}
\hline Plaintext & Key & Ciphertext \\
\hline 0000_0000_0000_0000 & $\begin{array}{l}0000 \text { 00000_0000_0000 } \\
0000 \text { 0000_0000_0000 }\end{array}$ & 3B07_DE72_9642_54AC \\
\hline 0000_0000_0000_0002 & 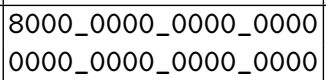 & C168_C69A_C195_845E \\
\hline FEDC_BA98_7654_3210 & $\begin{array}{l}\text { 0123_4567_89AB_CDEF } \\
0123 \text { 44567_89AB_CDEF }\end{array}$ & D9DF_068F_5993_8882 \\
\hline
\end{tabular}

